\title{
Induced energy-momentum tensor of a Dirac field in 2D de Sitter QED
}

\author{
Manizheh Botshekananfard๑* and Ehsan Bavarsad๑ $\odot^{\dagger}$ \\ Department of Physics, University of Kashan, 8731753153, Kashan, Iran
}

(Received 27 November 2019; accepted 24 March 2020; published 22 April 2020)

\begin{abstract}
We compute the expectation value of the energy-momentum tensor in the in-vacuum state of the quantized Dirac field coupled to a uniform electric field background on the Poincare patch of the twodimensional de Sitter spacetime $\left(\mathrm{dS}_{2}\right)$. The adiabatic regularization scheme is applied to remove the ultraviolet divergencies from the expressions. Then we obtain a finite result for the induced energymomentum tensor that varies continuously under a continuous change of the electric field strength and the Dirac field mass. We find that the off-diagonal components of the induced energy-momentum tensor vanish. The absolute values of the diagonal components increase as the electric field increases and decrease as the Dirac field mass increases except in the very near vicinity of their zero points. We derive the trace anomaly of the induced energy-momentum tensor, which agrees precisely with the trace anomaly derived earlier in the literature. We have discussed the backreaction of the induced energy-momentum tensor on the gravitational field of the $\mathrm{dS}_{2}$.
\end{abstract}

DOI: 10.1103/PhysRevD.101.085011

\section{INTRODUCTION}

The theory of renormalization of the quantum electrodynamics (QED) in Minkowski spacetime is well established. Generally, in a curved spacetime and especially in the case of the de Sitter spacetime $(\mathrm{dS})$, there is no unique vacuum state to compute the expectation values of the quantities. Furthermore, the additional divergences are caused by the gravitational interactions. In order to establish the theory of renormalization in curved spacetime various methods have been developed; see [1,2] for introduction. Perhaps one of the most efficient methods for regularizing the expectation values of the quantities is the adiabatic subtraction method. This method is based on the approximation that the creation of a particle with the frequency much larger than the energy scale determined by the curvature of the spacetime can be neglected. Hence, the adiabatic expansion is a power series of the spacetime curvature. Specifically, in the case of a Friedmann-Lemaitre-Robertson-Walker (FLRW) spacetime with a spatially flat metric the number time derivatives of the scale factor determines the adiabatic order. Adiabatic subtraction process starts from considering a Wentzel-KramersBrillouin (WKB) type solution for the under consideration mode equation and proceed to construction of the Fock

\footnotetext{
*m.botshekananfard@gmail.com

bavarsad@kashanu.ac.ir
}

Published by the American Physical Society under the terms of the Creative Commons Attribution 4.0 International license. Further distribution of this work must maintain attribution to the author(s) and the published article's title, journal citation, and DOI. Funded by SCOAP ${ }^{3}$. space of the state vectors to compute the expectation values of the quantities; see [1,2] for details. Adiabatic regularization was introduced by Parker to obtain a finite expectation value of the particle number in an expanding universe [3]. After this original work, adiabatic regularization generalized and applied for various types of quantities and particles. Using adiabatic regularization scheme, the energymomentum tensor of a scalar field has been computed in spatially flat [4-13] and closed [14] FLRW type universes, anisotropic spacetimes [15,16], and conformal coupling case [17]. The effective Lagrangian and the energymomentum tensor of a scalar field in dS have been computed in Ref. [18] using the dimensional regularization method. Cosmological applications motivated the authors of $[19,20]$ to compute the energy-momentum tensor of the created scalar particles in a four dimensional de Sitter spacetime $\left(\mathrm{dS}_{4}\right)$. In [19], the finite energy-momentum tensor has been defined as difference of the expectation values in the invacuum and out-vacuum states; and the author found the decay of the effective cosmological constant due to the Hawking effect. In [20], a novel renormalization technique has been applied to compute the energy-momentum tensor, and the authors found that for a massive noninteracting scalar field quantum corrections may lead to a superacceleration phase where the Hubble constant increase.

With the use of covariant point-splitting regularization approach, finite and unambiguous energy-momentum tensor of a scalar field has been calculated in a spatially flat FLRW spacetime [21,22] and dS [23], which are in agrement with the results obtained by applying dimensional regularization technique [18]. In [24], it was proven that the adiabatic and point-splitting regularization methods are 
equivalent in FLRW type spacetimes. The method of dimensional regularization is restricted only to special spacetimes which can be generalized to $n$ complex dimensions in a meaningful way, and the associated field equations can be solved explicitly $[23,24]$. In fact the point-splitting regularization is known to be the unique prescription (up to counterterms) that satisfies a list of desired properties, namely, locality, covariance, and appropriate continuity under variations of the coupling parameters and the metric; see, e.g., Ref. [25]. Although the point-splitting method can be applied to any spacetime, it deals with difficult calculations even in comparatively simple case of FLRW type spacetimes [23,24]. It is emphasized, the method of adiabatic regularization which deals with a mode-by-mode subtraction process is especially convenient for problems in which numerical calculations must be performed [1]. Hence, from a cosmological point of view which necessitate the use of numerical methods, adiabatic regularization is desirable. These facts illustrate the importance of the adiabatic regularization approach and motivate us to consider this method in this paper. In all the works [3-17] the adiabatic regularization method developed and applied for the case of a scalar field. The adiabatic expansion for the case of a Dirac field has been introduced in [26] and further details provided in [27]. The energy-momentum tensor of the Dirac field in a spatially flat FLRW spacetime has been regularized using the adiabatic subtraction prescription [28]; see also [2931]. As proved in [32] this method of regularization gives the same result as the DeWitt-Schwinger point-splitting regularization, when applied to the energy-momentum tensor of the Dirac field in a spatially flat FLRW universe.

In this paper, we purpose to go beyond considering only a scalar or fermion field in a curved spacetime background, by adding an electromagnetic gauge field to this picture. More specifically, we wish to consider a Dirac field coupled to a uniform electric field background in a dS. Creation of pairs in a strong electric field background in the flat spacetime is a well-known nonperturbative phenomenon in quantum field theory which is referred to as the Schwinger effect [33-35]; see [36,37] for introduction. The possibility of existing strong electromagnetic fields in the early universe [38] motivates studying the Schwinger effect in the cosmological context [39]; see [40] for a recent review and references. Due to the cosmological event horizon dS emits particles [10,11,19,41-50] which is known as the Gibbons-Hawking radiation [51,52]. In a dS, by using semiclassical approaches the Schwinger effect for scalar [53-64] and Dirac [65-70] fields has been investigated which may amplifies the Gibbons-Hawking radiation. Perturbative amplitude for production of scalar [71-75] and Dirac [76-78] pairs in the presence of an electric field on the dS has been studied and the authors found that the pair production is significant only when the expansion parameter is large compered to the mass of the field. Recently, the effect of a magnetic field on the Schwinger effect in the $\mathrm{dS}_{4}$ has been investigated [79]; see also [80-86], and it has been shown that the pair production is enhanced in the strong magnetic field regime. The Schwinger effect during inflation has been investigated using various approaches in Refs. [87-96]. To provide further evidence for the ER = EPR conjecture, the holographic Schwinger effect in dS was studied in Ref. [97].

As noted in [53,55], the in-vacuum state of the quantum fields in $\mathrm{dS}$ has the Hadamard form, i.e., the ultraviolet divergencies in the expectation values of the quantities which are calculated in this state, behaves similar to what is expected in the flat spacetime. Hence, it is a preferred vacuum state in study of renormalization theory in $\mathrm{dS}$. In the pioneering work [55], the Schwinger effect and the invacuum expectation value of the induced current of a scalar field in a $\mathrm{dS}_{2}$ has been investigated. The authors found that in the strong electric field regime, i.e., when the electric field is sufficiently large compered to the Ricci scalar curvature and the scalar field mass, the current agrees with the semiclassical result [53]. A notable feature of the current was found in the weak electric field regime for a small enough scalar field mass compered to the Hubble constant, where the current is inversely proportional to the electric field which leads to a phenomenon of infrared hyperconductivity. These remarkable results motivate the study of the induced current for both scalar and fermion fields in various dimensions of dS. The Schwinger effect and the adiabatic regularized induced current of scalar field in a $\mathrm{dS}_{4}$ has been investigated in [56], and particularly the phenomenon of infrared hyperconductivity was reported. In order to compare the results, the vacuum expectation value of the induced current was renormalized using pointsplitting method in [98] which completely agrees with the results of [56]. In [62], those results for the current have been derived by using the uniform asymptotic approximation method. The Schwinger effect in a general $D$-dimensional dS and, as an example of odd dimensions, the induced current of the scalar field in the three dimensional dS have been investigated in [57] which a period of the infrared hyperconductivity phenomenon was observed. Furthermore, the authors of [79] considered a uniform magnetic field with conserved flux parallel to a constant energy density electric field in the $\mathrm{dS}_{4}$ and found that the density of states are proportional to the magnetic field, consequently the scalar Schwinger effect is enhanced in the sufficiently large magnetic field. They also showed that in the sufficiently weak magnetic field the infrared hyperconductivity phenomenon occurs in the induced current of the scalar field. For the case of Dirac field the Schwinger effect and induced current of the created pairs have been investigated in $\mathrm{dS}_{2}$ [67] and $\mathrm{dS}_{4}$ [68]. Contrary to the scalar field case, no infrared hyperconductivity phenomenon has been observed in the induced fermionic current. Analyzing the induced current of both the scalar $[56,98]$ and the Dirac 
[68] fields in $\mathrm{dS}_{4}$ reveals a period of the negative current where the current flows in opposite direction of the electric field background weaker than a threshold value depending on the field mass. The negative current leads to the antiscreening effect which increases the magnitude of the electric field background. Obviously, the infrared hyperconductivity and the negative current phenomena are contrary to physical intuition. In attempts to address these peculiarities, a new renormalization condition defined for the scalar and Dirac induced currents in $\mathrm{dS}_{4}$ which was named maximal subtraction [99]. The maximal subtraction scheme defines the asymptotic value of the renormalized vacuum expectation value equal to that of obtained from the semiclassical approaches. Following this scheme the infrared hyperconductivity period is removed, however a phase of the negative current would be present. Recently, in [100] all the contributions to the regularized induced current in $\mathrm{dS}_{4}$ have been clarified. In all the works $[56,57,67,68]$ adiabatic subtraction scheme has been applied to regularize the induced current. In de Sitter spacetime with any dimension, the energy-momentum tensor of the semiclassical Schwinger scalars has been computed in the heavy scalar field [57] and the strong electric field [101] regimes; the authors found a decay of the Hubble constant due to the pair creation. The adiabatic regularized in-vacuum state expectation value of the trace of the induced energymomentum tensor for a charged scalar field coupled to a uniform electric field background in three [102] and four [103] dimensional dS has been studied. In [103], it was shown that gravitational backreaction effects of the trace of the induced energy-momentum tensor in the semiclassical regime leads to a decay of the Hubble constant, whereas in the infrared regime a superacceleration phenomenon occurs, i.e., the Hubble constant increases. The method of adiabatic subtraction for regularization of both cases scalar and spinor QED in an expanding universe has been extensively studied in Refs. [104-107]. In [108], a suitable adiabatic expansion has been introduced to regularize the energy-momentum tensor and helicity of the gauge fields coupled to a pseudoscalar field in a spatially flat FLRW spacetime. In this paper our main goals are the investigation of the induced energy-momentum tensor of the Dirac field in de Sitter QED and further exploring the validity of the adiabatic subtraction regularization. Hence, we consider a massive charged Dirac field coupled to a uniform electric field background in a $\mathrm{dS}_{2}$. To regularize the expectation value of the energy-momentum tensor in the in-vacuum state we adopt the second order adiabatic expansion of the appropriate counterterms.

The paper is structured as follows. In Sec. II, we briefly introduce our model. In Sec. III, we compute the expectation value of the energy-momentum tensor and regularize it. We examine the regularized energy-momentum tensor and discuses its consequences in Sec. IV. Eventually, the conclusions are presented in Sec. V.

\section{THE MODEL}

We consider a massive Dirac field coupled to a uniform electric field with constant energy density on the Poincaré patch of $\mathrm{dS}_{2}$. We assume the electric and gravitational fields as classical backgrounds which are not affected by the presence of the Dirac field. The half of $\mathrm{dS}_{2}$ can be represented as a spatially flat FLRW spacetime

$d s^{2}=d t^{2}-e^{2 H t} d \mathrm{x}^{2}, \quad t \in(-\infty, \infty), \quad \mathrm{x} \in \mathbb{R}$,

where $t$ is the proper time and $H$ is the Hubble constant. By using the transformation

$$
\tau=-\frac{1}{H} e^{-H t}, \quad \tau \in(-\infty, 0)
$$

the metric (1) can be expressed in a manifestly conformally flat form

$$
d s^{2}=\Omega^{2}(\tau)\left(d \tau^{2}-d \mathrm{x}^{2}\right), \quad \Omega(\tau)=-\frac{1}{H \tau} .
$$

In order to have a uniform electric field with a constant energy density in the metric (3), we choose the electromagnetic vector potential to be

$$
A_{\mu}(\tau)=\frac{E}{H^{2} \tau} \delta_{\mu}^{1}
$$

where $E$ is a constant.

The QED action for the Dirac field $\psi(x)$, with mass $m$ and charge $e$ which is coupled to the electromagnetic vector potential (4) in the $d S_{2}$ with metric (3) can be written as

$$
\begin{aligned}
S= & \int d^{2} x \sqrt{-g}\left\{\frac{i}{2} \bar{\psi}(x) \Gamma^{\mu} \mathcal{D}_{\mu} \psi(x)-\frac{i}{2} \bar{\psi}(x) \overleftarrow{\mathcal{D}_{\mu}} \Gamma^{\mu} \psi(x)\right. \\
& -m \bar{\psi}(x) \psi(x)\}
\end{aligned}
$$

where $g$ is the determinant of the metric, and $\Gamma^{\mu}$ are the Dirac gamma matrices in the $\mathrm{dS}_{2}$. The metric tensor of the spacetime $g_{\mu \nu}$, can be expressed in terms of the Minkowski metric $\eta_{a b}$, by using the tetrad coefficients $e^{a}{ }_{\mu}$,

$$
g_{\mu \nu}(\tau)=e^{a}{ }_{\mu}(\tau) e^{b}{ }_{\nu}(\tau) \eta_{a b} .
$$

We choose the tetrad in the gauge

$$
e_{\mu}^{a}(\tau)=\Omega(\tau) \delta_{\mu}^{a},
$$

then, the Dirac gamma matrices in the Weyl representation are given by

$$
\Gamma^{0}=\Omega^{-1}(\tau) \sigma_{1}, \quad \Gamma^{1}=\Omega^{-1}(\tau) i \sigma_{2},
$$


where $\sigma_{1}, \sigma_{2}$, and $\sigma_{3}$ are Pauli matrices. In this representation the adjoint Dirac field $\bar{\psi}(x)$, is defined

$$
\bar{\psi}(x)=\psi^{\dagger}(x) \sigma_{1} .
$$

The gauge covariant derivatives can be expressed as

$$
\mathcal{D}_{\mu}=\partial_{\mu}+B_{\mu}+i e A_{\mu}, \quad \overleftarrow{\mathcal{D}_{\mu}}=\overleftarrow{\partial_{\mu}}-B_{\mu}-i e A_{\mu}
$$

where the spin connection $B_{\mu}$, is given by

$$
B_{\mu}=-\frac{\dot{\Omega}}{2 \Omega} \sigma_{3} \delta_{\mu}^{1}
$$

where we use the over dot to denote the derivative with respect to the conformal time $\tau$.

The Dirac equation follows from the action (5),

$$
\left[i \Gamma^{\mu}\left(\partial_{\mu}+B_{\mu}+i e A_{\mu}\right)-m\right] \psi(x)=0 .
$$

By using the fact that the Dirac Eq. (12) is invariant under spatial translations, we can write $\psi(x)$ as decomposed twocomponent spinor

$$
\psi(x)=\psi(\tau, \mathrm{x})=e^{i k \mathrm{x}}\left(\begin{array}{c}
U_{1}(\tau) \\
U_{2}(\tau)
\end{array}\right) .
$$

Substituting the decomposed form of the spinor (13) into the Dirac Eq. (12), and by using Eqs. (4), (8), and (11) we arrive to a system of coupled deferential equations

$$
\begin{gathered}
i \dot{U}_{1}(\tau)+q(\tau) U_{1}(\tau)+i \frac{\dot{\Omega}(\tau)}{2 \Omega(\tau)} U_{1}(\tau)-m \Omega(\tau) U_{2}(\tau)=0 \\
i \dot{U}_{2}(\tau)-q(\tau) U_{2}(\tau)+\frac{i \dot{\Omega}(\tau)}{2 \Omega(\tau)} U_{2}(\tau)-m \Omega(\tau) U_{1}(\tau)=0
\end{gathered}
$$

where we have defined

$$
q(\tau)=k+e A_{1}(\tau)
$$

The system of coupled first order linear deferential equations (14) can be converted into an equivalent two single second order linear deferential equations

$$
\begin{aligned}
& \ddot{U}_{1}(\tau)+\left(\omega_{k}^{2}(\tau)-i q(\tau)\left(\frac{\dot{q}(\tau)}{q(\tau)}-\frac{\dot{\Omega}(\tau)}{\Omega(\tau)}\right)+\left(\frac{\ddot{\Omega}(\tau)}{2 \Omega(\tau)}-\frac{3 \dot{\Omega}^{2}(\tau)}{4 \Omega^{2}(\tau)}\right)\right) U_{1}(\tau)=0, \\
& \ddot{U}_{2}(\tau)+\left(\omega_{k}^{2}(\tau)+i q(\tau)\left(\frac{\dot{q}(\tau)}{q(\tau)}-\frac{\dot{\Omega}(\tau)}{\Omega(\tau)}\right)+\left(\frac{\ddot{\Omega}(\tau)}{2 \Omega(\tau)}-\frac{3 \dot{\Omega}^{2}(\tau)}{4 \Omega^{2}(\tau)}\right)\right) U_{2}(\tau)=0,
\end{aligned}
$$

where the conformal time dependent frequency is given by

$$
\omega(\tau)=+\sqrt{q^{2}(\tau)+m^{2} \Omega^{2}(\tau)} .
$$

The solutions of the Dirac Eqs. (16) with the desired asymptotic forms at early times $\tau \rightarrow-\infty$, and late times $\tau \rightarrow 0$, which describe the in and out-vacuum states, respectively, have been obtained in Ref. [67]. Since, we wish to compute the expectation value of the energymomentum tensor in the Hadamard in-vacuum state, we take only the solutions of Eqs. (16) with the desired asymptotic behavior at early times. Hence, the components of the normalized positive frequency mode spinor are given by [67]

$$
\begin{aligned}
U_{1 k}(\tau)= & -i \mu e^{\frac{i \pi \kappa}{2}} W_{\kappa-\frac{1}{2}, \gamma}(-2 i p) \Theta(k) \\
& +e^{-\frac{i \pi \kappa}{2}} W_{-\kappa+\frac{1}{2}, \gamma}(-2 i p) \Theta(-k), \\
U_{2 k}(\tau)= & e^{\frac{i \pi \kappa}{2}} W_{\kappa+\frac{1}{2}, \gamma}(-2 i p) \Theta(k) \\
& -i \mu e^{-\frac{i \pi \kappa}{2}} W_{-\kappa-\frac{1}{2}, \gamma}(-2 i p) \Theta(-k),
\end{aligned}
$$

and, the components of the normalized negative frequency mode spinor are obtained [67]

$$
V_{1 k}(\tau)=e^{\frac{i \pi \kappa}{2}} W_{-\kappa+\frac{1}{2}, \gamma}(2 i p) \Theta(k)-i \mu e^{-\frac{i \pi \kappa}{2}} W_{\kappa-\frac{1}{2}, \gamma}(2 i p) \Theta(-k),
$$

$$
\begin{aligned}
V_{2 k}(\tau)= & -i \mu e^{\frac{i \pi k}{2}} W_{-\kappa-\frac{1}{2}, \gamma}(2 i p) \Theta(k) \\
& +e^{-\frac{i \pi \kappa}{2}} W_{\kappa+\frac{1}{2}, \gamma}(2 i p) \Theta(-k),
\end{aligned}
$$

where $W$ is the Whittaker function [109], and the normalized step function has been defined as

$$
\Theta(k)=\sqrt{\frac{H}{2|k|}} \times \begin{cases}1 & k>0, \\ 0 & k<0 .\end{cases}
$$

We have defined the dimensionless variables

$$
\begin{aligned}
p=-|k| \tau, & \mu=\frac{m}{H}, & \lambda=\frac{e E}{H^{2}}, \\
r=\frac{k}{|k|}, & \kappa=-i \lambda, & \gamma=i \sqrt{\mu^{2}+\lambda^{2}} .
\end{aligned}
$$


The normalized mode spinor components which are given by Eqs. (18)-(21), may be represented in the doublet form

$$
U_{k}(\tau)=\left(\begin{array}{c}
U_{1 k}(\tau) \\
U_{2 k}(\tau)
\end{array}\right), \quad V_{k}(\tau)=\left(\begin{array}{c}
V_{1 k}(\tau) \\
V_{2 k}(\tau)
\end{array}\right) .
$$

Then, it can be shown that these doublets satisfy the relation

$$
U_{k}(\tau) U_{k}^{\dagger}(\tau)+V_{k}(\tau) V_{k}^{\dagger}(\tau)=\Omega^{-1}(\tau) \mathbb{I},
$$

where $\mathbb{I}$ denotes the $2 \times 2$ dimensional unit matrix.

The set of complete orthonormal mode spinors (24) determines the in-vacuum state $|0\rangle$ which is annihilated by acting the operators $a_{k}$ for a fermion and $b_{k}$ for an antifermion with the comoving momentum $k$, as

$$
a_{k}|0\rangle=b_{k}|0\rangle=0, \quad \forall k .
$$

Then, the Fock space of state vectors is built by successive acting the creation operators $a_{k}^{\dagger}$ and $b_{k}^{\dagger}$ for fermions and antifermions, respectively. To quantize the Dirac field $\psi(x)$, we impose the anticommutation relations

$$
\left\{a_{k}, a_{k^{\prime}}^{\dagger}\right\}=\left\{b_{k}, b_{k^{\prime}}^{\dagger}\right\}=(2 \pi) \delta\left(k-k^{\prime}\right) .
$$

Then, the quantized Dirac field operator $\psi(x)$, can be written as

$$
\psi(x)=\int_{-\infty}^{+\infty} \frac{d k}{(2 \pi)}\left[a_{k} U_{k}(\tau) e^{i k \mathrm{x}}+b_{k}^{\dagger} V_{k}(\tau) e^{-i k \mathrm{x}}\right] .
$$

\section{REGULARIZATION OF ENERGY- MOMENTUM TENSOR}

The energy-momentum tensor of the Dirac field is defined by variation of the action (5) with respect to variation of the inverse metric $\delta g^{\mu \nu}$, or more precisely

$$
T_{\mu \nu}(x)=\frac{2}{\sqrt{|g|}} \frac{\delta S}{\delta g^{\mu \nu}} .
$$

After some algebra, definition (29) yields the expression for the energy-momentum tensor of the Dirac field coupled to the uniform electric field background in the $\mathrm{dS}_{2}$, as

$$
T_{\mu \nu}(x)=\frac{i}{4}\left(\bar{\psi} \Gamma_{\mu} \mathcal{D}_{\nu} \psi+\bar{\psi} \Gamma_{\nu} \mathcal{D}_{\mu} \psi-\bar{\psi} \overleftarrow{\mathcal{D}_{\nu}} \Gamma_{\mu} \psi-\bar{\psi} \overleftarrow{\mathcal{D}_{\mu}} \Gamma_{\nu} \psi\right)
$$

\section{A. In-vacuum state expectation values}

By considering the Dirac field $\psi(x)$, as the quantum operator which is given by Eq. (28) and using the relations (26) and (27), we arrive at the following expressions for the in-vacuum state expectation values of the energymomentum tensor components. The timelike component is given by

$$
\left\langle 0\left|T_{00}(x)\right| 0\right\rangle=-\frac{H^{2} \Omega^{2}(\tau)}{2 \pi} \Re \sum_{r= \pm 1} \int_{0}^{\Lambda} \frac{d p}{p}\left[p^{2}-\mu^{2}(p-\lambda r) e^{\pi \lambda r}\left|W_{i \lambda r-\frac{1}{2}, \gamma}(2 i p)\right|^{2}+i \mu^{2} e^{-\pi \lambda r} W_{-i \lambda r-\frac{1}{2}, \gamma}(2 i p) W_{i \lambda r+\frac{1}{2}, \gamma}(-2 i p)\right],
$$

where $\Re$ denotes the real part of the expression. For the spacelike component we have

$$
\left\langle 0\left|T_{11}(x)\right| 0\right\rangle=-\frac{H^{2} \Omega^{2}(\tau)}{2 \pi} \sum_{r= \pm 1} \int_{0}^{\Lambda} \frac{d p}{p}\left[p^{2}-\mu^{2}(p-\lambda r) e^{\pi \lambda r}\left|W_{i \lambda r-\frac{1}{2}, \gamma}(2 i p)\right|^{2}\right],
$$

and the symmetric off-diagonal component is obtained

$$
\left\langle 0\left|T_{01}(x)\right| 0\right\rangle=\frac{H^{2} \Omega^{2}(\tau)}{2 \pi} \lambda \Lambda .
$$

To regularize the ultraviolet divergencies in the momentum integrals of Eqs. (31)-(33), we have considered cutoff $K$ for the comoving momentum $k$. Accordingly, cutoff $\Lambda=-K \tau$ is defined for the dimensionless physical momentum $p$; see definitions in Eq. (23).

To evaluate the momentum integrals involving the Whittaker functions in Eqs. (31) and (32), we follow the procedure that introduced in Refs. [55,56] for computing the induced current of the scalar field in a $\mathrm{dS}_{2}$ and $\mathrm{dS}_{4}$, respectively. This method of integration has also been used for computing the induced current of the Dirac field in a $\mathrm{dS}_{2}$ [67] and $\mathrm{dS}_{4}$ [68]. The appropriate Mellin-Barnes integral representation [109] of the Whittaker function is given by

$$
\begin{aligned}
W_{\kappa, \gamma}(z)= & e^{-\frac{z}{2}} \int_{-i \infty}^{+i \infty} \frac{d s}{2 \pi i} \frac{\Gamma\left(\frac{1}{2}+\gamma+s\right) \Gamma\left(\frac{1}{2}-\gamma+s\right) \Gamma(-\kappa-s)}{\Gamma\left(\frac{1}{2}+\gamma-\kappa\right) \Gamma\left(\frac{1}{2}-\gamma-\kappa\right)} z^{-s}, \\
& \frac{1}{2} \pm \gamma-\kappa \neq 0,-1,-2, \ldots
\end{aligned}
$$


which is vialed for arbitrary values of the phase of the argument in the domain $|\operatorname{ph}(z)|<3 \pi / 2$. The condition is that the contour of integration must be arranged in a way that the chain of poles of $\Gamma\left(\frac{1}{2}+\gamma+s\right) \Gamma\left(\frac{1}{2}-\gamma+s\right)$ lies a part from the chain of poles $\Gamma(-\kappa-s)$. By means of Eq. (34) and the theorem of residues, we then obtain the final results

$$
\begin{aligned}
\left\langle 0\left|T_{00}(x)\right| 0\right\rangle= & \Omega^{2}(\tau) \frac{H^{2}}{4 \pi}\left[-2 \Lambda^{2}-2 \mu^{2} \log (2 \Lambda)-2 \lambda^{2}-\mu^{2}-i \gamma \sinh (2 \pi \lambda) \csc (2 \pi \gamma)+i \lambda+2 \gamma \lambda \sinh (2 \pi \lambda) \csc (2 \pi \gamma)\right. \\
& \left.+\mu^{2}(1+i \sinh (2 \pi \lambda) \csc (2 \pi \gamma)) \psi(i \lambda+\gamma)+\mu^{2}(1-i \sinh (2 \pi \lambda) \csc (2 \pi \gamma)) \psi(i \lambda-\gamma)\right],
\end{aligned}
$$

and

$$
\begin{aligned}
\left\langle 0\left|T_{11}(x)\right| 0\right\rangle= & \Omega^{2}(\tau) \frac{H^{2}}{4 \pi}\left[-2 \Lambda^{2}+2 \mu^{2} \log (2 \Lambda)-2 \lambda^{2}-\mu^{2}+i \gamma \sinh (2 \pi \lambda) \csc (2 \pi \gamma)-i \lambda+2 \gamma \lambda \sinh (2 \pi \lambda) \csc (2 \pi \gamma)\right. \\
& \left.-\mu^{2}(1+i \sinh (2 \pi \lambda) \csc (2 \pi \gamma)) \psi(i \lambda+\gamma)-\mu^{2}(1-i \sinh (2 \pi \lambda) \csc (2 \pi \gamma)) \psi(i \lambda-\gamma)\right],
\end{aligned}
$$

where $\log$ is the natural logarithm function and $\psi$ denotes the digamma function which is defined by the first derivative of the logarithm of the Gamma function; see, e.g., [109].

\section{B. Adiabatic regularization}

To remove the ultraviolet divergencies from the expectation values given by Eqs. (33), (35), and (36) we apply the adiabatic subtraction scheme. This method has been used to regularize the induced fermionic current in $\mathrm{dS}_{2}$ [67] and $\mathrm{dS}_{4}$ [68]. To find the appropriate counterterms, we perform the same procedure as used in [67]. As usual in the literature, we assume that the electromagnetic vector potential and energy-momentum tensor in the two dimensional spacetime to be of adiabatic order zero and two, respectively. We begin by considering a WKB type solution for the Dirac equations (16) as

$$
\mathcal{U}_{a}(\tau)=N_{a} \exp \left[-i \int^{\tau}\left(X_{a}\left(\tau^{\prime}\right)+i Y_{a}\left(\tau^{\prime}\right)\right) d \tau^{\prime}\right]
$$

where $a=1,2$ is the spinor index, $N_{a}$ is a normalization coefficient, and the real functions $X_{a}$ and $Y_{a}$ are required to satisfy equations

$$
Y_{a}(\tau)=(-1)^{a} \frac{q(\tau)}{2 X_{a}(\tau)}\left(\frac{\dot{q}(\tau)}{q(\tau)}-\frac{\dot{\Omega}(\tau)}{\Omega(\tau)}\right)-\frac{1}{2} \frac{d}{d \tau} \log \left(X_{a}(\tau)\right),
$$

$X_{a}^{2}(\tau)=Y_{a}^{2}(\tau)+\dot{Y}_{a}(\tau)+\omega^{2}(\tau)+\frac{\ddot{\Omega}(\tau)}{2 \Omega(\tau)}-\frac{3 \dot{\Omega}^{2}(\tau)}{4 \Omega^{2}(\tau)}$.

Inserting the function $Y_{a}$ from Eq. (38) into Eq. (37), we find that $\mathcal{U}_{a}$ can be expressed as

$$
\mathcal{U}_{a}(\tau)=\frac{N_{a}}{\sqrt{X_{a}(\tau)}} \exp \left[\int^{\tau}\left(-i X_{a}\left(\tau^{\prime}\right)+(-1)^{a} \frac{q\left(\tau^{\prime}\right)}{2 X_{a}\left(\tau^{\prime}\right)}\left(\frac{\dot{q}\left(\tau^{\prime}\right)}{q\left(\tau^{\prime}\right)}-\frac{\dot{\Omega}\left(\tau^{\prime}\right)}{\Omega\left(\tau^{\prime}\right)}\right)\right) d \tau^{\prime}\right] .
$$

Then, substituting $\mathcal{U}_{a}$ from Eq. (40) into the decoupled Dirac Eqs. (16) yields an algebraic equation for the function $X_{a}$, which exactly is

$$
\begin{aligned}
X_{a}^{2}(\tau)-\omega^{2}(\tau)= & -\frac{1}{2 X_{a}}\left(\ddot{X}_{a}-(-1)^{a} \ddot{q}\right)+\frac{1}{4 X_{a}^{2}}\left(3 \dot{X}_{a}^{2}+\dot{q}^{2}\right)-(-1)^{a} \frac{\dot{X}_{a} q}{X_{a}^{2}}\left(\frac{\dot{q}}{q}-\frac{\dot{\Omega}}{\Omega}\right) \\
& -\frac{q^{2}}{4 X_{a}^{2}} \frac{\dot{\Omega}}{\Omega}\left(2 \frac{\dot{q}}{q}-\frac{\dot{\Omega}}{\Omega}\right)-(-1)^{a} \frac{q}{2 X_{a}}\left(\frac{\dot{\Omega}}{\Omega} \frac{\dot{q}}{q}-\frac{\dot{\Omega}^{2}}{\Omega^{2}}+\frac{\ddot{\Omega}}{\Omega}\right)-\frac{3 \dot{\Omega}^{2}}{4 \Omega^{2}}+\frac{\ddot{\Omega}}{2 \Omega} .
\end{aligned}
$$

The remaining undetermined factor in the adiabatic mode spinor (37) is the normalization constant $N_{a}$. We will show below that the counterterms are independent of $N_{a}$. We constrain the components of the adiabatic mode spinor (37) to obey the normalization condition

$$
\left|\mathcal{U}_{1}(\tau)\right|^{2}+\left|\mathcal{U}_{2}(\tau)\right|^{2}=\Omega^{-1}(\tau)
$$

Following the adiabatic regularization method, the set of counterterms are constructed from the expectation values of the energy-momentum tensor components (30) in the adiabatic vacuum sate which is described by the solutions (37). Such expressions can be written as 


$$
\mathcal{T}_{00}=\int_{-K}^{+K} \frac{d k}{(2 \pi)}\left[\frac{q(\tau)\left(\left|\mathcal{U}_{1}\right|^{2}-\left|\mathcal{U}_{2}\right|^{2}\right)-m \Omega(\tau)\left(\mathcal{U}_{1} \mathcal{U}_{2}^{*}+\mathcal{U}_{1}^{*} \mathcal{U}_{2}\right)}{\left|\mathcal{U}_{1}\right|^{2}+\left|\mathcal{U}_{2}\right|^{2}}\right]
$$

and, for the spacelike component

$$
\mathcal{T}_{11}=\int_{-K}^{+K} \frac{d k}{(2 \pi)} q(\tau)\left[\frac{\left|\mathcal{U}_{1}\right|^{2}-\left|\mathcal{U}_{2}\right|^{2}}{\left|\mathcal{U}_{1}\right|^{2}+\left|\mathcal{U}_{2}\right|^{2}}\right]
$$

where $K$ is an ultraviolet momentum cutoff. The symmetric off-diagonal component finally is obtained

$$
\mathcal{T}_{01}=-\frac{1}{2} \int_{-K}^{+K} \frac{d k}{(2 \pi)} q(\tau)=\frac{H^{2} \Omega^{2}(\tau)}{2 \pi} \lambda \Lambda .
$$

Defining the ratio of the spinor components

$$
\mathcal{R}=\frac{\mathcal{U}_{2}}{\mathcal{U}_{1}}
$$

one can rewrite Eqs. (43) and (44) as

$$
\begin{gathered}
\mathcal{T}_{00}=\int_{-K}^{+K} \frac{d k}{(2 \pi)}\left[\frac{q(\tau)\left(1-|\mathcal{R}|^{2}\right)-m \Omega(\tau)\left(\mathcal{R}+\mathcal{R}^{*}\right)}{1+|\mathcal{R}|^{2}}\right] \\
\mathcal{T}_{11}=\int_{-K}^{+K} \frac{d k}{(2 \pi)} q(\tau)\left[\frac{1-|\mathcal{R}|^{2}}{1+|\mathcal{R}|^{2}}\right]
\end{gathered}
$$

The expressions for $\mathcal{U}_{1}$ and $\mathcal{U}_{2}$ can be read from Eq. (40) with $a=1$ and $a=2$, respectively. Substituting these expressions into the coupled Dirac Eqs. (14), we then obtain

$$
\mathcal{R}=\frac{1}{\Omega m}\left[X_{1}+q-\frac{i\left(\dot{X}_{1}+\dot{q}\right)}{2 X_{1}}+\frac{i \dot{\Omega}\left(X_{1}+q\right)}{2 X_{1} \Omega}\right],
$$

where $X_{1}$ satisfies Eq. (41) with $a=1$. By virtue of Eqs. (43)-(49) the counterterms are independent of the normalization constants $N_{a}$, as mentioned above.

We now return to Eq. (41), to find a solution for the function $X_{1}$. Obviously, terms appearing on the right side of Eq. (41) are of two higher adiabatic order than those on the left side. By using the fact that the conformal time dependent frequency $\omega$ [see Eq. (17)] is of zero adiabatic order, at the lowest adiabatic order the function $X_{a}$ is obtained

$$
X_{a}^{(0)}(\tau)=\omega(\tau)
$$

where the superscript denotes the adiabatic order. Then, up to the second order the adiabatic expansion of $X_{a}$ is constructed iteratively and is given by

$$
\begin{aligned}
X_{a}^{(2)}(\tau)= & \omega(\tau)-\frac{1}{4 \omega^{2}}\left(\ddot{\omega}-(-1)^{a} \ddot{q}\right)+\frac{1}{8 \omega^{3}}\left(3 \dot{\omega}^{2}+\dot{q}^{2}\right)-(-1)^{a} \frac{\dot{\omega} q}{2 \omega^{3}}\left(\frac{\dot{q}}{q}-\frac{\dot{\Omega}}{\Omega}\right) \\
& -\frac{q^{2}}{8 \omega^{3}} \frac{\dot{\Omega}}{\Omega}\left(2 \frac{\dot{q}}{q}-\frac{\dot{\Omega}}{\Omega}\right)-(-1)^{a} \frac{q}{4 \omega^{2}}\left(\frac{\dot{\Omega}}{\Omega} \frac{\dot{q}}{q}-\frac{\dot{\Omega}^{2}}{\Omega^{2}}+\frac{\ddot{\Omega}}{\Omega}\right)-\frac{1}{2 \omega}\left(\frac{3 \dot{\Omega}^{2}}{4 \Omega^{2}}-\frac{\ddot{\Omega}}{2 \Omega}\right) .
\end{aligned}
$$

Hence, the adiabatic expansions of $X_{1}$, up the order of zero and two are read from Eqs. (50) and (51) with $a=1$, respectively. Then, substituting the second adiabatic order expansion of the expression on the right side of Eq. (49) into Eqs. (47) and (48), we finally obtain

$$
\begin{aligned}
& \mathcal{T}_{00}^{(2)}=\Omega^{2}(\tau) \frac{H^{2}}{2 \pi}\left[-\Lambda^{2}-\mu^{2} \log (2 \Lambda)+\frac{1}{12}+\mu^{2} \log (\mu)-\frac{\mu^{2}}{2}-\lambda^{2}+\frac{\lambda^{2}}{6 \mu^{2}}\right] \\
& \mathcal{T}_{11}^{(2)}=\Omega^{2}(\tau) \frac{H^{2}}{2 \pi}\left[-\Lambda^{2}+\mu^{2} \log (2 \Lambda)-\frac{1}{12}-\mu^{2} \log (\mu)-\frac{\mu^{2}}{2}-\lambda^{2}-\frac{\lambda^{2}}{6 \mu^{2}}\right] .
\end{aligned}
$$

Subtracting the second adiabatic order counterterms, given by Eqs. (45), (52), and (53) from the corresponding original expressions for the in-vacuum expectation values which are given by Eqs. (33), (35), and (36), leads to the regularized induced energy-momentum tensor

$$
\begin{aligned}
T_{00}= & \left\langle 0\left|T_{00}(x)\right| 0\right\rangle-\mathcal{T}_{00}^{(2)} \\
= & \Omega^{2}(\tau) \frac{H^{2}}{4 \pi}\left[-\frac{1}{6}-\mu^{2} \log \left(\mu^{2}\right)-\frac{\lambda^{2}}{3 \mu^{2}}-i \gamma \sinh (2 \pi \lambda) \csc (2 \pi \gamma)+i \lambda+2 \gamma \lambda \sinh (2 \pi \lambda) \csc (2 \pi \gamma)\right. \\
& \left.+\mu^{2}(1+i \sinh (2 \pi \lambda) \csc (2 \pi \gamma)) \psi(i \lambda+\gamma)+\mu^{2}(1-i \sinh (2 \pi \lambda) \csc (2 \pi \gamma)) \psi(i \lambda-\gamma)\right],
\end{aligned}
$$

and 


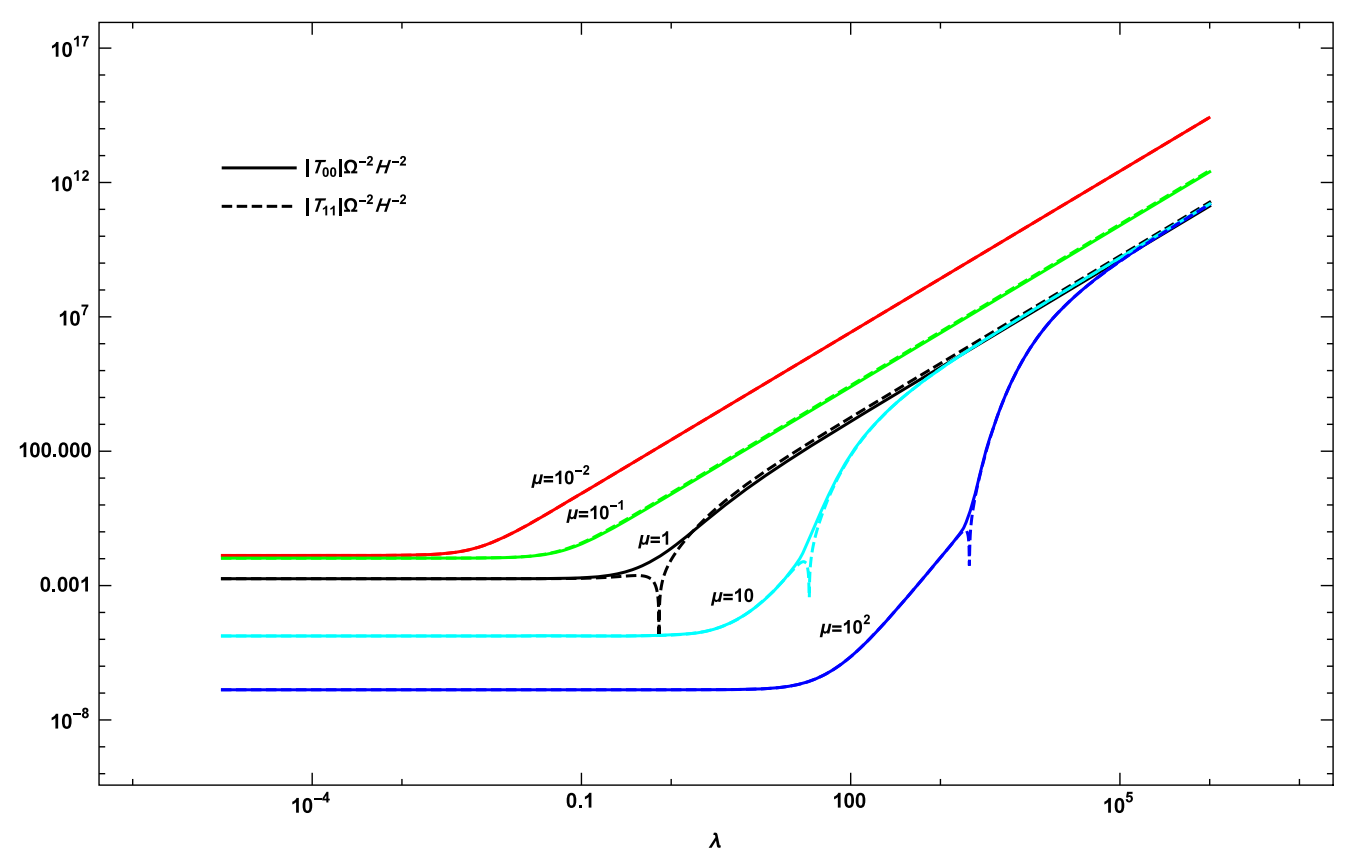

FIG. 1. The normalized absolute values of the induced energy-momentum tensor components $\left|T_{00}\right| \Omega^{-2} H^{-2}$, in solid line and $\left|T_{11}\right| \Omega^{-2} H^{-2}$ in dashed line are plotted as functions of the normalized electric field $\lambda=e E / H^{2}$, for different values of the normalized Dirac field mass $\mu=m / H$. The scales are logarithmic on both axes.

$$
\begin{aligned}
T_{11}= & \left\langle 0\left|T_{11}(x)\right| 0\right\rangle-\mathcal{T}_{11}^{(2)} \\
= & \Omega^{2}(\tau) \frac{H^{2}}{4 \pi}\left[\frac{1}{6}+\mu^{2} \log \left(\mu^{2}\right)+\frac{\lambda^{2}}{3 \mu^{2}}+i \gamma \sinh (2 \pi \lambda) \csc (2 \pi \gamma)-i \lambda+2 \gamma \lambda \sinh (2 \pi \lambda) \csc (2 \pi \gamma)\right. \\
& \left.-\mu^{2}(1+i \sinh (2 \pi \lambda) \csc (2 \pi \gamma)) \psi(i \lambda+\gamma)-\mu^{2}(1-i \sinh (2 \pi \lambda) \csc (2 \pi \gamma)) \psi(i \lambda-\gamma)\right] .
\end{aligned}
$$

The important result is that the symmetric off-diagonal component of the regularized induced energy-momentum tensor vanishes

$$
T_{01}=\left\langle 0\left|T_{01}(x)\right| 0\right\rangle-\mathcal{T}_{01}=0 .
$$

\section{EXAMINATION OF THE INDUCED ENERGY-MOMENTUM TENSOR}

In this section, we examine the regularized energymomentum tensor which is given by Eqs. (54)-(56). In Figs. 1 and 2, we have logarithmically plotted the absolute value of $T_{00}$ (54) and $T_{11}$ (55) as functions of the parameters electric field $\lambda$ and Dirac field mass $\mu$, respectively. Figure 1 shows that for a fixed value of $\mu$, the absolute value of $T_{00}$ increases analytically as $\lambda$ increases. For a fixed value of $\lambda$, as $\mu$ increases smoothly from zero, $T_{00}$ increases analytically from large negative values passes through zero and near this point attains a maximum, then falls continuously to zero. For the case of $T_{11}$, Fig. 1 shows that for a fixed value of $\mu \lesssim 1$, the absolute value of $T_{11}$ is an analytic increasing function of $\lambda$. For a fixed value of $\mu \gtrsim 1$, as $\lambda$ increases smoothly from zero, $T_{11}$ increases analytically from negative values and passes through zero, then continuously tends to infinity. For a fixed value of $\lambda$, as $\mu$ increases smoothly from zero, $T_{11}$ decrease analytically from large positive values passes through zero and near this point attains a minimum, then continuously approaches zero. It should be noted that in Figs. 1 and 2, the points at which $T_{00}$ or $T_{11}$ become zero appear as sharp singularities in the logarithmic plots of the absolute values of these expressions, i.e., $\left|T_{00}\right|$ and $\left|T_{11}\right|$. Thus, the components of the induced energy-momentum tensor are analytic and continuous functions of the parameters $\lambda$ and $\mu$. The magnitudes of the diagonal components of the induced energy-momentum tensor, i.e., $\left|T_{00}\right|$ and $\left|T_{11}\right|$ increase as the electric field increases and decrease as the mass increases except in the very near vicinity of their zero points.

\section{A. Asymptotic behaviors}

We now analyze the induced energy-momentum tensor in the limiting values of the electric field and the Dirac field 


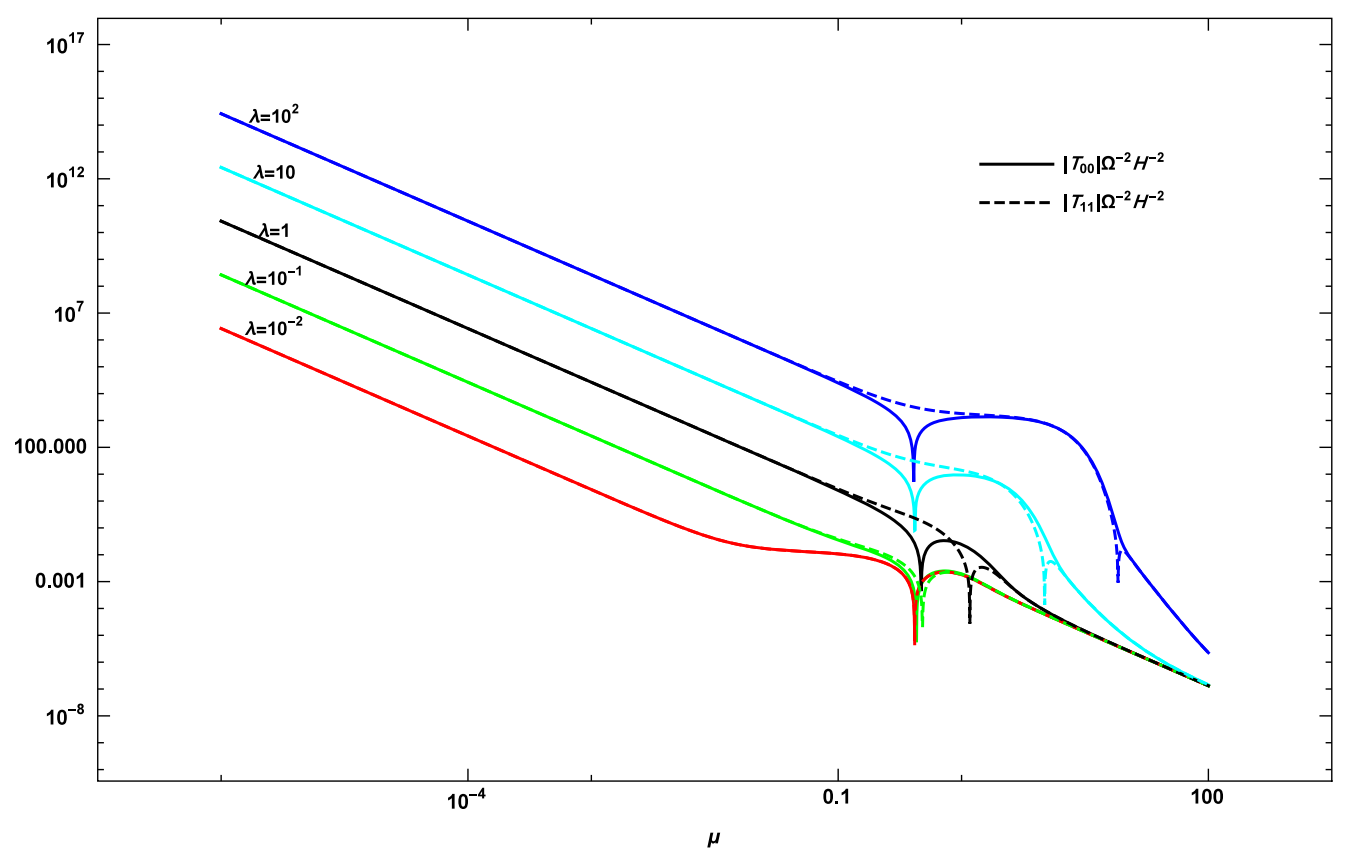

FIG. 2. The normalized absolute values of the induced energy-momentum tensor components $\left|T_{00}\right| \Omega^{-2} H^{-2}$, in solid line and $\left|T_{11}\right| \Omega^{-2} H^{-2}$ in dashed line are plotted as functions of the normalized Dirac field mass $\mu=m / H$, for different values of the normalized electric field $\lambda=e E / H^{2}$. The scales are logarithmic on both axes.

mass, to find the explicit asymptotic behaviors shown in Figs. 1 and 2.

\section{Strong electric field}

In the strong electric field regime, the asymptotic behavior of the induced energy-momentum tensor is obtained by expanding $T_{00}$ (54) and $T_{11}(55)$ in a Taylor series about $\lambda=\infty$ with fixed $\mu$. We then obtain the leading order terms as

$$
\begin{aligned}
& T_{00} \simeq \Omega^{2}(\tau) \frac{H^{2} \lambda^{2}}{2 \pi}\left(e^{-\frac{\pi \mu^{2}}{\lambda}}-\frac{1}{6 \mu^{2}}\right), \\
& T_{11} \simeq \Omega^{2}(\tau) \frac{H^{2} \lambda^{2}}{2 \pi}\left(e^{-\frac{\pi \mu^{2}}{\lambda}}+\frac{1}{6 \mu^{2}}\right) .
\end{aligned}
$$

\section{Weak electric field}

In the weak electric field regime, the asymptotic behavior of the induced energy-momentum tensor is obtained by expanding $T_{00}$ (54) and $T_{11}$ (55) in a Taylor series about $\lambda=0$. For the case of a light Dirac field $\mu \ll 1$, we obtain the leading order terms

$$
\begin{aligned}
T_{00} & =-T_{11} \\
& \simeq-\Omega^{2}(\tau) H^{2}\left(\frac{1}{24 \pi}+\frac{\lambda^{2}}{12 \pi \mu^{2}}+\frac{\mu^{2}}{4 \pi} \log \left(\mu^{2}\right)+\frac{\gamma_{\mathrm{E}} \mu^{2}}{2 \pi}\right),
\end{aligned}
$$

where $\gamma_{\mathrm{E}}=0.577 \cdots$ is Euler's constant. And for the case of a heavy Dirac field $\mu \gg 1$, the leading order terms are given by

$$
T_{00} \simeq \Omega^{2}(\tau) H^{2}\left(\frac{1}{240 \pi \mu^{2}}+4 \lambda^{2} \mu e^{-2 \pi \mu}-\frac{\lambda^{2}}{3 \mu} e^{-2 \pi \mu}\right)
$$

$$
T_{11} \simeq \Omega^{2}(\tau) H^{2}\left(\frac{-1}{240 \pi \mu^{2}}+\frac{\lambda^{2}}{3 \mu} e^{-2 \pi \mu}\right)
$$

\section{Heavy Dirac field}

For the case of a heavy Dirac field, we can expand $T_{00}$ (54) and $T_{11}$ (55) in a Taylor series about $\mu=\infty$ with fixed $\lambda$. We find that the expressions

$$
T_{00} \simeq \Omega^{2}(\tau) H^{2}\left(\frac{1}{240 \pi \mu^{2}}+\frac{\lambda \mu}{\pi} e^{-2 \pi \mu}\right),
$$

$$
T_{11} \simeq \Omega^{2}(\tau) H^{2}\left(\frac{-1}{240 \pi \mu^{2}}+\frac{\lambda}{12 \pi \mu}\left(1-4 \lambda^{2}\right) e^{-2 \pi \mu}\right),
$$

well approximate the behavior of the diagonal components of the induced energy-momentum tensor as long as $\lambda \ll \mu$.

\section{B. Trace of the induced energy-momentum tensor}

The trace of the induced energy-momentum tensor reads from Eqs. (54) and (55), 


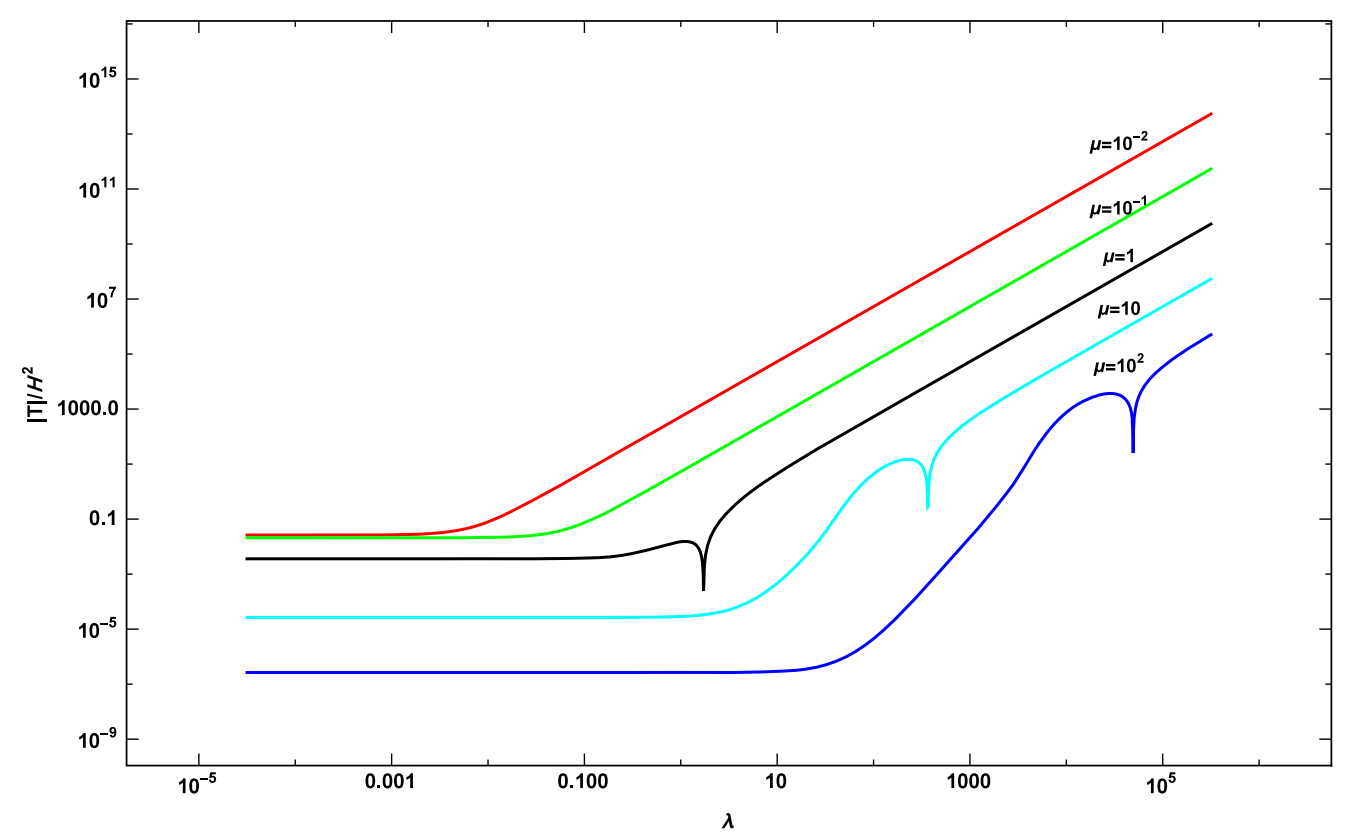

FIG. 3. The normalized absolute value of the trace of the induced energy-momentum tensor $|T| / H^{2}$, is plotted as function of the normalized electric field $\lambda=e E / H^{2}$, for different values of the normalized Dirac field mass $\mu=m / H$. The scales are logarithmic on both axes.

$$
\begin{aligned}
T= & g^{\mu \nu} T_{\mu \nu} \\
= & \frac{H^{2}}{2 \pi}\left[-\frac{1}{6}-\mu^{2} \log \left(\mu^{2}\right)-\frac{\lambda^{2}}{3 \mu^{2}}-i \gamma \sinh (2 \pi \lambda) \csc (2 \pi \gamma)+i \lambda\right. \\
& +\mu^{2}(1+i \sinh (2 \pi \lambda) \csc (2 \pi \gamma)) \psi(i \lambda+\gamma) \\
& \left.+\mu^{2}(1-i \sinh (2 \pi \lambda) \csc (2 \pi \gamma)) \psi(i \lambda-\gamma)\right]
\end{aligned}
$$

whose absolute value has been logarithmically plotted in Fig. 3. For a fixed value of $\mu \lesssim 1$, as $\lambda$ increases from zero, $T$ monotonically goes to large negative values, thus as shown in Fig. 3 its absolute value, $|T|$, is an increasing function of $\lambda$. For a fixed value of $\mu \gtrsim 1$, as $\lambda$ increases from zero, $T$ increases from a positive small value and attains a maximum then it decreases and passes through zero when $\lambda$ reaches a specific value $\lambda_{*}$ and then analytically goes to large negative values. In this case, at the point $\lambda=\lambda_{*}$ at which $T$ becomes zero, a sharp singular behavior appears in the logarithmic plot of the absolute value of $T$ in Fig. 3 . Hence, the trace of the induced energy-momentum tensor is an analytic and continuous function of the parameters $\lambda$ and $\mu$, whose magnitude increase as the electric field increases and decrease as the mass increases except in the very near vicinity of its zero point.

We emphasize, for the case of a light Dirac field $\mu \lesssim 1$, the trace is negative $T<0$. For the case of a heavy Dirac field $\mu \gtrsim 1$, the precise value of $\lambda_{*}$ is evaluated by solving the equation

$$
T\left(\mu, \lambda_{*}\right)=0
$$

For $\lambda<\lambda_{*}$ the trace is positive $T>0$, whereas for $\lambda>\lambda_{*}$ the trace is negative $T<0$. To find the behavior of $\lambda_{*}$ against the Dirac field mass parameter $\mu$, at which the evaluation was made, we present Fig. 4 which shows that $\lambda_{*}$ increases as $\mu$ increases.

Before concluding, we would like to point out some consequences of the trace of the induced energymomentum tensor, namely, the trace anomaly and gravitational backreaction.

\section{Trace anomaly}

The trace anomaly of the induced energy-momentum tensor is derived by taking the limit of $\lambda \rightarrow 0$ and $\mu \rightarrow 0$ in the expression (64), which yields

$$
T=-\frac{R}{24 \pi},
$$

where $R=2 H^{2}$ is the Ricci scalar curvature of $\mathrm{dS}_{2}$. This result agrees precisely with the well-known trace anomaly [110] of a Dirac field in the two-dimensional de Sitter spacetime.

\section{Gravitational backreaction}

In Ref. [111], the backreaction effect of the induced Dirac field current on the electromagnetic field background in $\mathrm{dS}_{2}$ has been studied. The main conclusion was that the backreaction of the fermion pair creation does not amplify the electric field background. In this section we wish to study the backreaction of the induced energy-momentum 


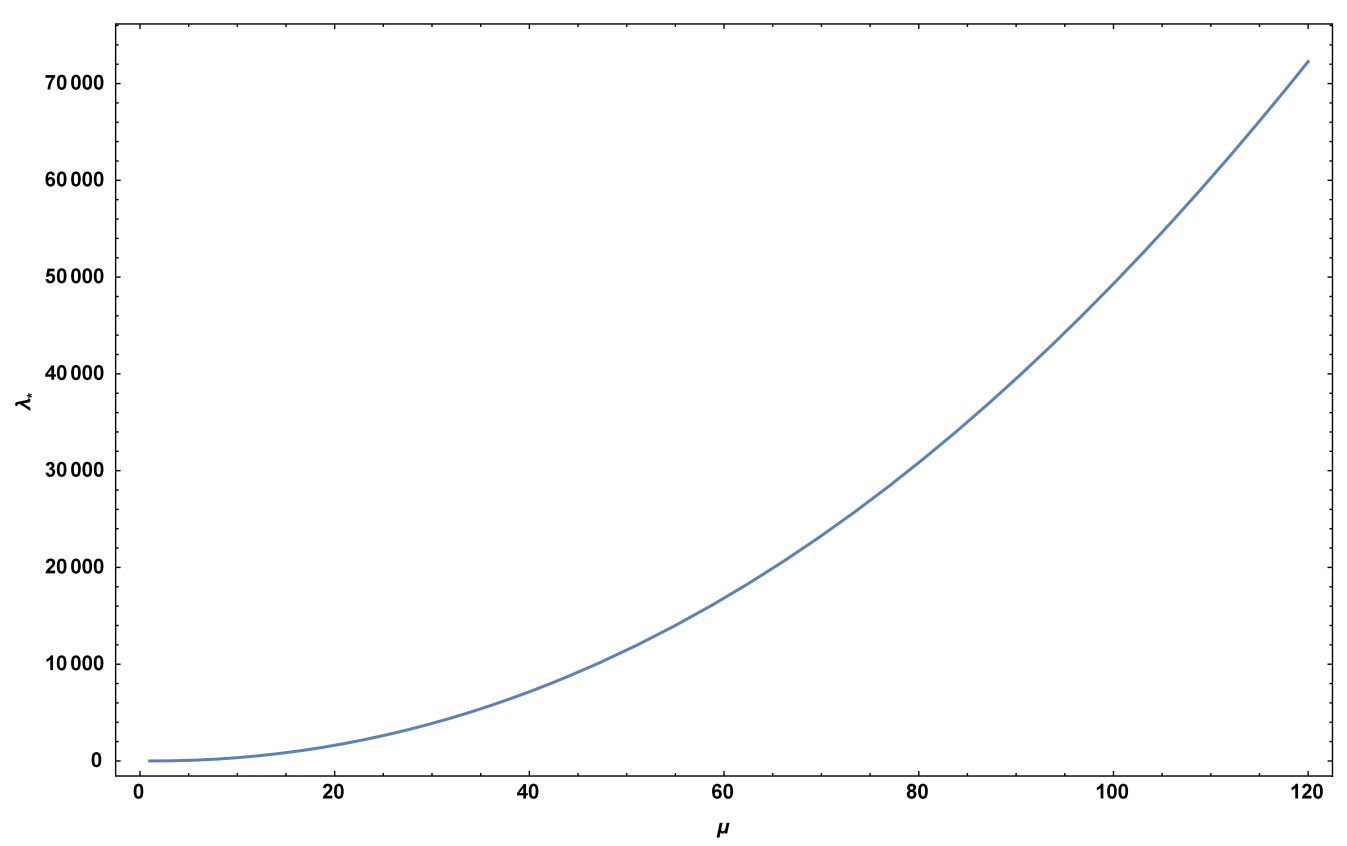

FIG. 4. The values of $\lambda_{*}$ are plotted against the normalized Dirac field mass $\mu$ at which the evaluation was made.

tensor on the gravitational field background. A natural way to do this is to use the Einstein gravitational field equations. However, as is well known, there is no Einstein gravity in two-dimensional spacetimes. Perhaps the most natural analogues of the Einstein gravitational field equations in two-dimensions is given by

$$
R-\Lambda=8 \pi G T
$$

where $R$ is the Ricci scalar curvature, $\Lambda$ is the cosmological constant, $G$ is Newton's gravitational constant in twodimensions and $T$ is the trace of the energy-momentum tensor; see, e.g., $[112,113]$.

In a $\mathrm{dS}_{2}$ the cosmological constant vanishes, hence we set $\Lambda=0$ in Eq. (67). In natural units, Newton's gravitational constant $G$ should be dimensionless in two-dimensional spacetimes. Hence in a $\mathrm{dS}_{2}$ the Newton's gravitational constant, in terms of the Planck mass $M_{\mathrm{P}}$ and the Hubble constant $H$, can be written naturally as $G=H^{2} / M_{\mathrm{P}}^{2}$. To explore the consequences of induced energy-momentum tensor, it is convent to define the time dependent Hubble parameter as

$$
H(\tau)=\Omega^{-2}(\tau) \frac{d \Omega(\tau)}{d \tau} .
$$

The pair creation begins from early times, $\tau \rightarrow-\infty$, where the Ricci scalar curvature is given by $R=2 H^{2}(\tau)$ and the induced energy-momentum tensor of the created pairs is negligible compared to the Planck mass; and eventually, finishes at late times, $\tau \rightarrow 0$, where the Ricci scalar curvature approaches zero, $R \approx 0$, and the induced energy-momentum tensor of the created pairs is given by Eqs. (54)-(56).
Therefore, in this picture the time scale of the pair creation is of order of the Hubble time, i.e., $\delta \tau \approx H^{-1}$, and variations of the Ricci scalar curvature, $\delta R$, and the trace of the induced energy-momentum tensor, $\delta T$, are given by

$$
\delta R=-R, \quad \delta T=T,
$$

where $T$ is given by Eq. (64). Then, variation of both sides of Eq. (67) leads to the evolution equation for the Hubble parameter

$$
\dot{H}(\tau)=-\frac{2 \pi H^{2}}{M_{\mathrm{P}}^{2}} T .
$$

Therefore, if $T>0$ then the Hubble constant decays. This condition holds for the case of heavy Dirac fields $\mu \gtrsim 1$ created by an electric field background with the strength $\lambda<\lambda_{*}$. Whereas, the Hubble constant amplifies and a superacceleration phenomenon with $\dot{H}>0$ occurs in the condition that $T<0$. This is the case for light Dirac fields $\mu \lesssim 1$ created by an unbounded electric field strength or creation of heavy quanta $\mu \gtrsim 1$ in an electric field with the strength $\lambda>\lambda_{*}$.

\section{CONCLUSION}

In this paper we have computed the in-vacuum state expectation value of the energy-momentum tensor of a Dirac field in a uniform electric field background on the Poincaré patch of $\mathrm{dS}_{2}$. As expected the expectation values in the Hadamard in-vacuum state acquire quadratic ultraviolet divergences; see Eqs. (33), (35), and (36). To obtain finite expressions, we have applied the adiabatic regularization scheme. Since the energy-momentum tensor is of second adiabatic order in two dimensions, we have 
constructed the set of the appropriate counterterms up to the second order of the adiabatic expansions; see Eqs. (45), (52), and (53). Hence, we reach our goal of driving the induced energy-momentum tensor in Eqs. (54)-(56). We have shown that the off-diagonal components of the induced energy-momentum tensor vanish. We have found that the magnitudes of the diagonal components of the induced energy-momentum tensor increase as the electric field increases and decrease as the mass increases except in the very near vicinity of their zero points; see Figs. 1 and 2. More precisely, these behaviors have been determined in Eqs. (57)-(63). We conclude that the components of the induced energy-momentum tensor are analytic and continuous functions of the electric field and Dirac field mass.

The trace of the induced energy-momentum tensor is given by Eq. (64), whose absolute value logarithmically is plotted in Fig. 3. We have found that the trace of the induced energy-momentum tensor is an analytic and continuous function of the parameters electric field and mass, whose magnitude increases as the electric field increases and decreases as the mass increases except in the very near vicinity of its zero point. The analytic behavior of the trace in the limiting values of the electric field and mass can be read from Eqs. (57)-(63). For the case of a light Dirac field $\mu \lesssim 1$, the trace is negative $T<0$. We have found that for the case of a heavy Dirac field $\mu \gtrsim 1$, the trace becomes zero at a specific value of $\lambda$, which is denoted by $\lambda_{*}$. The sign of the trace changes at $\lambda=\lambda_{*}$. For $\lambda<\lambda_{*}$ the trace is positive $T>0$, whereas for $\lambda>\lambda_{*}$ the trace is negative $T<0$. Figure 4 shows that $\lambda_{*}$ increases as the Dirac field mass increases. We have shown that the induced energymomentum tensor acquires the trace anomaly [see Eq. (66)] which precisely agrees with the trace anomaly derived earlier in the literature for a Dirac field in $\mathrm{dS}_{2}$.

In Sec. IV D, we have briefly discussed the evolution of the Hubble constant caused by the induced energy-momentum tensor; see the final result in Eq. (70). We have found that creation of heavy Dirac fields $\mu \gtrsim 1$ in an electric field whose strength is bounded as $\lambda<\lambda_{*}$ leads to a decay of the Hubble constant due to the positive trace of the induced energy-momentum tensor. Whereas, creation of light Dirac fields $\mu \lesssim 1$ in an electric field with unbounded strength or heavy Dirac fields $\mu \gtrsim 1$ in an electric field with the strength $\lambda>\lambda_{*}$ amplifies the Hubble constant and a superacceleration phenomenon with $\dot{H}>0$ occurs due to the negative trace of the induced energy-momentum tensor.

\section{ACKNOWLEDGMENTS}

E. B. is supported by the University of Kashan Grant No. 890243/1.
[1] L. E. Parker and D. Toms, Quantum Field Theory in Curved Spacetime: Quantized Field and Gravity (Cambridge University Press, Cambridge, England, 2009).

[2] N. D. Birrell and P. C. W. Davies, Quantum Fields in Curved Space (Cambridge University Press, Cambridge, England, 1984).

[3] L. Parker, Phys. Rev. Lett. 21, 562 (1968).

[4] S. A. Fulling and L. Parker, Ann. Phys. (N.Y.) 87, 176 (1974).

[5] L. Parker and S. A. Fulling, Phys. Rev. D 9, 341 (1974).

[6] T. S. Bunch, J. Phys. A 13, 1297 (1980).

[7] S. Habib, C. Molina-Paris, and E. Mottola, Phys. Rev. D 61, 024010 (1999).

[8] D. L. Nacir and F. D. Mazzitelli, Phys. Rev. D 76, 024013 (2007).

[9] D. Lopez Nacir, F. D. Mazzitelli, and C. Simeone, J. Phys. A 40, 6895 (2007)

[10] P. R. Anderson and E. Mottola, Phys. Rev. D 89, 104038 (2014).

[11] P. R. Anderson and E. Mottola, Phys. Rev. D 89, 104039 (2014).

[12] Y. Zhang, X. Ye, and B. Wang, Sci. China Phys. Mech. Astron. 63, 250411 (2020).

[13] Y. Zhang, B. Wang, and X. Ye, arXiv:1909.13010.
[14] P. R. Anderson and L. Parker, Phys. Rev. D 36, 2963 (1987).

[15] B. L. Hu and L. Parker, Phys. Rev. D 17, 933 (1978); 17, 3292(E) (1978).

[16] D. Lopez Nacir and F. D. Mazzitelli, Phys. Rev. D 78, 044001 (2008).

[17] S. A. Fulling, L. Parker, and B. L. Hu, Phys. Rev. D 10, 3905 (1974); Phys. Rev. D 11, 1714(E) (1975).

[18] J. S. Dowker and R. Critchley, Phys. Rev. D 13, 3224 (1976).

[19] E. Mottola, Phys. Rev. D 31, 754 (1985).

[20] T. Markkanen and A. Rajantie, J. High Energy Phys. 01 (2017) 133.

[21] P. C. W. Davies, S. A. Fulling, S. M. Christensen, and T. S. Bunch, Ann. Phys. (N.Y.) 109, 108 (1977).

[22] T. S. Bunch and P. C.W. Davies, J. Phys. A 11, 1315 (1978).

[23] T. S. Bunch and P. C. W. Davies, Proc. R. Soc. A 360, 117 (1978).

[24] N. D. Birrell, Proc. R. Soc. A 361, 513 (1978).

[25] S. Hollands and R. M. Wald, Commun. Math. Phys. 223, 289 (2001).

[26] A. Landete, J. Navarro-Salas, and F. Torrentí, Phys. Rev. D 88, 061501(R) (2013). 
[27] A. Landete, J. Navarro-Salas, and F. Torrentí, Phys. Rev. D 89, 044030 (2014).

[28] A. del Rio, J. Navarro-Salas, and F. Torrenti, Phys. Rev. D 90, 084017 (2014).

[29] S. Ghosh, Phys. Rev. D 91, 124075 (2015).

[30] S. Ghosh, Phys. Rev. D 93, 044032 (2016).

[31] A. del Rio, A. Ferreiro, J. Navarro-Salas, and F. Torrenti, Phys. Rev. D 95, 105003 (2017).

[32] A. del Río and J. Navarro-Salas, Phys. Rev. D 91, 064031 (2015).

[33] J. Schwinger, Phys. Rev. 82, 664 (1951).

[34] F. Sauter, Z. Phys. 69, 742 (1931).

[35] W. Heisenberg and H. Euler, Z. Phys. 98, 714 (1936).

[36] F. Gelis and N. Tanji, Prog. Part. Nucl. Phys. 87, 1 (2016).

[37] G. V. Dunne, Heisenberg-Euler Effective Lagrangians: Basics and Extensions, edited by M. Shifman et al., From fields to strings Vol. 1 (World Scientific Publishing, Singapore, 2005), pp. 445-522.

[38] R. Durrer and A. Neronov, Astron. Astrophys. Rev. 21, 62 (2013).

[39] J. Martin, Lect. Notes Phys. 738, 193 (2008).

[40] S. P. Kim, arXiv:1905.13439.

[41] R. Bousso, A. Maloney, and A. Strominger, Phys. Rev. D 65, 104039 (2002).

[42] A. M. Polyakov, Nucl. Phys. B797, 199 (2008).

[43] A. M. Polyakov, Nucl. Phys. B834, 316 (2010).

[44] G. Mahajan and T. Padmanabhan, Gen. Relativ. Gravit. 40, 709 (2008).

[45] J. Haro and E. Elizalde, J. Phys. A 41, 372003 (2008).

[46] E. Greenwood, D. C. Dai, and D. Stojkovic, Phys. Lett. B 692, 226 (2010).

[47] R. Dabrowski and G. V. Dunne, Phys. Rev. D 90, 025021 (2014).

[48] S. P. Kim, Phys. Rev. D 88, 044027 (2013).

[49] A. Havare, T. Yetkin, M. Korunur, and K. Sogut, Nucl. Phys. B682, 457 (2004).

[50] M. R. Tanhayi, Int. J. Mod. Phys. D 24, 1550052 (2015).

[51] S. W. Hawking, Commun. Math. Phys. 43, 199 (1975); 46, 206(E) (1976).

[52] G. W. Gibbons and S. W. Hawking, Phys. Rev. D 15, 2738 (1977).

[53] J. Garriga, Phys. Rev. D 49, 6343 (1994).

[54] J. Garriga, Phys. Rev. D 49, 6327 (1994).

[55] M. B. Fröb, J. Garriga, S. Kanno, M. Sasaki, J. Soda, T. Tanaka, and A. Vilenkin, J. Cosmol. Astropart. Phys. 04 (2014) 009.

[56] T. Kobayashi and N. Afshordi, J. High Energy Phys. 10 (2014) 166.

[57] E. Bavarsad, C. Stahl, and S. S. Xue, Phys. Rev. D 94, 104011 (2016).

[58] R. G. Cai and S. P. Kim, J. High Energy Phys. 09 (2014) 072.

[59] S. P. Kim, Int. J. Mod. Phys. D 25, 1645005 (2016).

[60] S. P. Kim and D. N. Page, Phys. Rev. D 78, 103517 (2008).

[61] S. P. Kim, Gravitation Cosmol. 20, 193 (2014).

[62] J. J. Geng, B. F. Li, J. Soda, A. Wang, Q. Wu, and T. Zhu, J. Cosmol. Astropart. Phys. 02 (2018) 018.

[63] R. Sharma and S. Singh, Phys. Rev. D 96, 025012 (2017).

[64] B. Hamil and M. Merad, Int. J. Mod. Phys. A 33, 1850177 (2018).
[65] V. M. Villalba, Phys. Rev. D 52, 3742 (1995).

[66] S. Haouat and R. Chekireb, Phys. Rev. D 87, 088501 (2013).

[67] C. Stahl, E. Strobel, and S. S. Xue, Phys. Rev. D 93, 025004 (2016).

[68] T. Hayashinaka, T. Fujita, and J. Yokoyama, J. Cosmol. Astropart. Phys. 07 (2016) 010.

[69] C. Stahl and S. Eckhard, AIP Conf. Proc. 1693, 050005 (2015).

[70] S. Haouat and R. Chekireb, arXiv:1504.08201.

[71] M. A. Băloi, Mod. Phys. Lett. A 29, 1450138 (2014).

[72] N. Nicolaevici, Mod. Phys. Lett. A 30, 1550046 (2015).

[73] C. Crucean and M. A. Băloi, Int. J. Mod. Phys. A 30, 1550088 (2015).

[74] M. A. Băloi, arXiv:1903.02029.

[75] C. Crucean and M. A. Băloi, Mod. Phys. Lett. A 31, 1650082 (2016).

[76] I. I. Cotăescu and C. Crucean, Phys. Rev. D 87, 044016 (2013).

[77] C. Cosmin, Phys. Rev. D 85, 084036 (2012).

[78] M. A. Băloi and C. Crucean, Int. J. Mod. Phys. A 32, 1750208 (2017).

[79] E. Bavarsad, S. P. Kim, C. Stahl, and S. S. Xue, Phys. Rev. D 97, 025017 (2018).

[80] E. Bavarsad, S. P. Kim, C. Stahl, and S. S. Xue, EPJ Web Conf. 168, 03002 (2018).

[81] S. Moradi, Mod. Phys. Lett. A 24, 1129 (2009).

[82] C. Crucean and M. A. Băloi, Phys. Rev. D 93, 044070 (2016).

[83] N. Nicolaevici and A. Farkas, Phys. Rev. D 95, 048501 (2017).

[84] C. Crucean and M. A. Băloi, Phys. Rev. D 95, 048502 (2017).

[85] M. A. Băloi, C. Crucean, and D. Popescu, Eur. Phys. J. C 78, 398 (2018).

[86] M. A. Băloi, D. Popescu, and C. Crucean, arXiv:1905.06561.

[87] C. Stahl, Nucl. Phys. B939, 95 (2019).

[88] W. Tangarife, K. Tobioka, L. Ubaldi, and T. Volansky, J. High Energy Phys. 02 (2018) 084.

[89] H. Kitamoto, Phys. Rev. D 98, 103512 (2018).

[90] M. Giovannini, Phys. Rev. D 97, 061301(R) (2018).

[91] W. Z. Chua, Q. Ding, Y. Wang, and S. Zhou, J. High Energy Phys. 04 (2019) 066.

[92] S. Shakeri, M. A. Gorji, and H. Firouzjahi, Phys. Rev. D 99, 103525 (2019).

[93] O. O. Sobol, E. V. Gorbar, M. Kamarpour, and S.I. Vilchinskii, Phys. Rev. D 98, 063534 (2018).

[94] O. O. Sobol, E. V. Gorbar, and S. I. Vilchinskii, Phys. Rev. D 100, 063523 (2019).

[95] E. V. Gorbar, A. I. Momot, O. O. Sobol, and S. I. Vilchinskii, Phys. Rev. D 100, 123502 (2019).

[96] V. Domcke, Y. Ema, and K. Mukaida, J. High Energy Phys. 02 (2020) 055.

[97] W. Fischler, P. H. Nguyen, J. F. Pedraza, and W. Tangarife, Phys. Rev. D 91, 086015 (2015).

[98] T. Hayashinaka and J. Yokoyama, J. Cosmol. Astropart. Phys. 07 (2016) 012.

[99] T. Hayashinaka and S. S. Xue, Phys. Rev. D 97, 105010 (2018). 
[100] M. Banyeres, G. Domènech, and J. Garriga, J. Cosmol. Astropart. Phys. 10 (2018) 023.

[101] E. Bavarsad and N. Margosian, J. Res. Many-Body Syst. 8, 1 (2018).

[102] E. Bavarsad and M. Mortezazadeh, Iran. J. Phys. Res. 18, 91 (2018).

[103] E. Bavarsad, S. P. Kim, C. Stahl, and S. S. Xue, arXiv: 1909.09319.

[104] A. Ferreiro and J. Navarro-Salas, Phys. Lett. B 792, 81 (2019).

[105] A. Ferreiro, J. Navarro-Salas, and S. Pla, Phys. Rev. D 98, 045015 (2018).

[106] A. Ferreiro and J. Navarro-Salas, Phys. Rev. D 97, 125012 (2018).
[107] J. F. Barbero G., A. Ferreiro, J. Navarro-Salas, and E. J. S. Villaseñor, Phys. Rev. D 98, 025016 (2018).

[108] M. Ballardini, M. Braglia, F. Finelli, G. Marozzi, and A. A. Starobinsky, Phys. Rev. D 100, 123542 (2019).

[109] F. W. J. Olver, D. W. Lozier, R. F. Boisvert, and C. W. Clark, NIST Handbook of Mathematical Functions (Cambridge University Press, Cambridge, England, 2010).

[110] M. J. Duff, Nucl. Phys. B125, 334 (1977).

[111] C. Stahl and S. S. Xue, Phys. Lett. B 760, 288 (2016).

[112] R. B. Mann, A. Shiekh, and L. Tarasov, Nucl. Phys. B341, 134 (1990).

[113] R. B. Mann, Gen. Relativ. Gravit. 24, 433 (1992). 